\title{
Analysis of the p53 Tumor Suppressor Gene by Pyrosequencing
}

BioTechniques 28:140-147 (January 2000)

\section{A. Ahmadian, J. Lundeberg, P. Nyrén, M. Uhlén and M. Ronaghi \\ The Royal Institute of Tech- nology, Stockholm, Sweden}

\section{INTRODUCTION}

Tumor suppressor genes involved in cancer development (2) are usually recessive, and in most cases, alterations occur in both alleles for inactivation (8). One such tumor suppressor gene is the p53 gene, which is reported to be the most commonly mutated gene in human cancers $(3,5)$. We have previously reported on the inactivation of both alleles of the $p 53$ gene by mutations and loss of heterozygosity in basal cell cancer and squamous cell carcinoma of the skin (9-11). In these samples, a predominant feature is inactivation by multiple mutations. Direct Sanger sequencing of PCR products will yield the consensus sequence of the target sample, but it will not yield information on which allele these mutations reside. To determine whether multiple mutations are located on one allele or separate alleles (biallelic inactivation), the cloning of PCR products, amplification of clones and re-sequencing of clones is necessary $(9,10,15)$.

Recently, Ronaghi et al. described an alternative technique for conventional DNA sequencing called pyrosequencing (14). Pyrosequencing uses natural nucleotides to monitor DNA synthesis and measure the absolute amount of nucleotide incorporation. This sequencing-by-synthesis method relies on the detection of $\mathrm{PP}_{\mathrm{i}}$ that is released upon nucleotide incorporation by using a four-enzyme mixture (Figure 1). The released $\mathrm{PP}_{\mathrm{i}}$ is converted to adenosine triphosphate (ATP) by ATP sulfurylase, which, in turn, is sensed by luciferase to generate a proportional amount of light. The light output can be recorded by a photon-detector device. Unreacted nucleotides are removed by apyrase to allow the cyclic addition of nucleotides to the reaction system (Figure 1). The technique's ability to sequence DNA obtained from PCR-amplified templates has been demonstrated $(12,13)$ and was recently improved by the addition of a single-stranded (ss) DNA-binding protein (unpublished data).

Here, we discuss the possibility of using the pyrosequencing technique for distinguishing genetic alterations in the p53 tumor suppressor gene. We also describe, quantification of the ratio between mutated and wild-type PCR-amplified fragments, as well as the ability to read nucleotide substitutions in exons 5 and 6 of the $p 53$ gene.

\section{MATERIALS AND METHODS}

\section{Microdissection, PCR Amplification and Conventional DNA Sequencing}

Microdissected biopsy samples were stained and analyzed under the microscope as described earlier $(4,11)$, and the stained cells were transferred to tubes containing $50 \mu \mathrm{L}$ PCR buffer [10 $\mathrm{mmol} / \mathrm{L}$ Tris- $\mathrm{HCl}$ (pH 8.3), $50 \mathrm{mmol} / \mathrm{L}$ $\mathrm{KCl}$. Cells were lysed by the addition of $2 \mu \mathrm{L}$ of freshly prepared proteinase $\mathrm{K}$ solution $(20 \mathrm{mg} / \mathrm{mL}$, dissolved in re-distilled water) at $56^{\circ} \mathrm{C}$ for $1 \mathrm{~h}$ (Roche Molecular Biochemical, Mannheim, Germany). Multiplex PCR amplification and solid-phase DNA sequencing of the p53 gene in exons 4-9 was performed as previously described $(1,6)$. In one case, exon 5 of the p53 gene was also sequenced by using Big Dye terminators 
on a $4 \%$ denaturing polyacrylamide gel for ABI PRISM 377XL (both from PE Biosystems, Foster City, CA, USA).

\section{Template Preparation for Pyrosequencing}

Biotinylated PCR products were immobilized onto streptavidin-coated super paramagnetic beads (Dynal AS, Oslo, Norway). The ssDNA was obtained by incubating the immobilized PCR product in $6 \mu \mathrm{L} 0.1 \mathrm{~mol} / \mathrm{L} \mathrm{NaOH}$ for 3-4 min. The supernatant was then neutralized by adding $3 \mu \mathrm{L} 0.2 \mathrm{~mol} / \mathrm{L}$ $\mathrm{HCl}$. The immobilized strand was resolved in $9 \mu \mathrm{L} \mathrm{H}_{2} \mathrm{O}$. Primers IMMp53EX5 (5'-GCTATCTGAGCAGCGCT-3') and SUPp53EX6 (5'-GGTGCCCTATGAGCCGCC-3') were used as pyrosequencing primers for the detection of the known mutations in exons 5 and 6, respectively (Figures 2, 3). For exon 5, we used the immobilized strand, and for exon 6, the eluted strand was used. One $\mu \mathrm{L} 10 \times$ annealing buffer $(100 \mathrm{mmol} / \mathrm{L}$ TA pH 7.75, 20 $\left.\mathrm{mmol} / \mathrm{L} \mathrm{MgAc}_{2}\right)$ and sequencing primer $(0.1 \mu \mathrm{mol} / \mathrm{L})$ were added to the single-stranded templates in a total volume of $10 \mu \mathrm{L}$. Hybridization was performed by incubation at $50^{\circ} \mathrm{C}$ for $2 \mathrm{~min}$ and then at room temperature for $5 \mathrm{~min}$.

\section{Pyrosequencing}

Pyrosequencing was performed at room temperature in a volume of $50 \mu \mathrm{L}$ on an automated single-tube pyrosequencer prototype (Pyrosequencing AB, Uppsala, Sweden). Briefly, 1/10 of a $50 \mu \mathrm{L}$ PCR (using 5 pmol PCR primers) was used for an assay. Then, $0.5 \mu \mathrm{g}$ ssDNA-binding protein (Amersham Pharmacia Biotech, Uppsala, Sweden) was added to primed target DNA. The obtained complex was added to the pyrosequencing reaction mixture that contained $10 \mathrm{U}$ exonuclease-deficient (exo-) Klenow DNA polymerase (Amersham Pharmacia Biotech), 40 $\mathrm{mU}$ apyrase (Sigma, St. Louis, MO, USA), 100 ng purified luciferase (BioThema, Dalarö, Sweden), $15 \mathrm{mU}$ recombinant ATP sulfurylase (7), 0.1 $\mathrm{mol} / \mathrm{L}$ Tris-acetate (pH 7.75), 0.5 $\mathrm{mmol} / \mathrm{L}$ EDTA, $5 \mathrm{mmol} / \mathrm{L}$ magnesium acetate, $0.1 \%$ bovine serum albumin (BioThema), $1 \mathrm{mmol} / \mathrm{L}$ dithiothreitol, 2 $\mu \mathrm{mol} / \mathrm{L}$ adenosine $5^{\prime}$ phosphosulfate
(APS) (Sigma), $0.4 \mathrm{mg} / \mathrm{mL}$ polyvinylpyrrolidone $(360000)$ and $100 \mu \mathrm{g} / \mathrm{mL}$ D-luciferin (BioThema). The sequencing procedure was carried out by stepwise elongation of the primer-strand after the sequential addition of $40 \mathrm{pmol}$ of the different deoxynucleoside triphosphates (Amersham Pharmacia Biotech) and the simultaneous degradation of nucleotides by apyrase.

\section{Determination of Detection Limit in Pyrosequencing}

To determine the detection limit for mutation signals, two oligonucleotide templates were constructed that corresponded to the investigated region in exon 5 of the p53 gene. The oligonucleotides differed in two positions that represent the wild-type sequence 
(5'-GCGCTGCCCCCACCATGAGCGCTGCTCAGATAGCGA-3') and the mutant variant $\left(5^{\prime}\right.$-GCGCTGCCCCCATAATGAGCGC GCTCAGATAGCGA-3') (underlined bases indicate the mutation sites). Four different ratios of wild-type/mutant (wt/mut) oligonucleotides, $0 \%, 25 \%, 50 \%$ and $100 \%$ mutant signal, respectively, were used for pyrosequencing (Figure 1).

\section{RESULTS}

In this study, we investigated the use of pyrosequencing in detecting polymorphic sequences. Initially, oligonucleotide templates were used in a model system. The technology was then applied to clinical samples for an analysis of mutations in the p53 tumor suppressor gene on non-melanoma skin cancer samples.

\section{Mutation Detection Limit in Pyrose- quencing}

To determine the detection limit of mutation signals using pyrosequencing, two oligonucleotide templates were constructed. The templates were designed to harbor the identical sequences as a clinical tumor sample with $\mathrm{CC}$ to AT substitutions in exon 5 of the $p 53$ gene (Figure 3). One oligonucleotide template contained the wild-type sequence and one contained the two mutations. The primed oligonucleotide templates with the wild-type and mutant sequence were mixed in proportions that resulted in a $50 \%$ mixture and a $75 \%$ wild-type to $25 \%$ mutant mixture, respectively. Pyrosequencing was performed by the iterative addition of $\mathrm{C}, \mathrm{G}$, $\mathrm{T}$ and $\mathrm{A}$ nucleotides. Each experiment was repeated at least three times, and the same results were obtained. Figure 2 illustrates the pyrosequencing data obtained using the different mixtures.

By evaluating the pyrosequencing raw data, it is possible to quantify the number of incorporated bases by following the peak heights. Figure 2B, I shows one base incorporation signal in the wild-type sequence for the first three bases (C, A and T). After the C, A and $\mathrm{T}$ signals, a peak twice as high is obtained that indicates a two-base incorporation (GG). The corresponding data for a homogenous mutant sequence is shown in Figure 2B, II and shows an incorporation of two $\mathrm{T} \mathrm{nu}-$ cleotides (instead of one), followed by incorporation of one A nucleotide. The two patterns (I and II) are easy to distinguish. By studying the 50/50 mixture and the $75 / 25$ mixture (Figure 2B, III and IV), it is obvious that a genetic heterogeneity exists when compared with the wild-type sequence. The nature of the variability can be analyzed by comparing the sum of incorporated bases over a defined region. For example, the third and fourth peak positions in the wild-type sequence correspond to three incorporated bases ( $\mathrm{T}$ and $\mathrm{G}$ ). In the mixture of $50 \%$ wild-type to $50 \%$ mutant (Figure 2B, III), a total of threebase incorporation is achieved by sum-

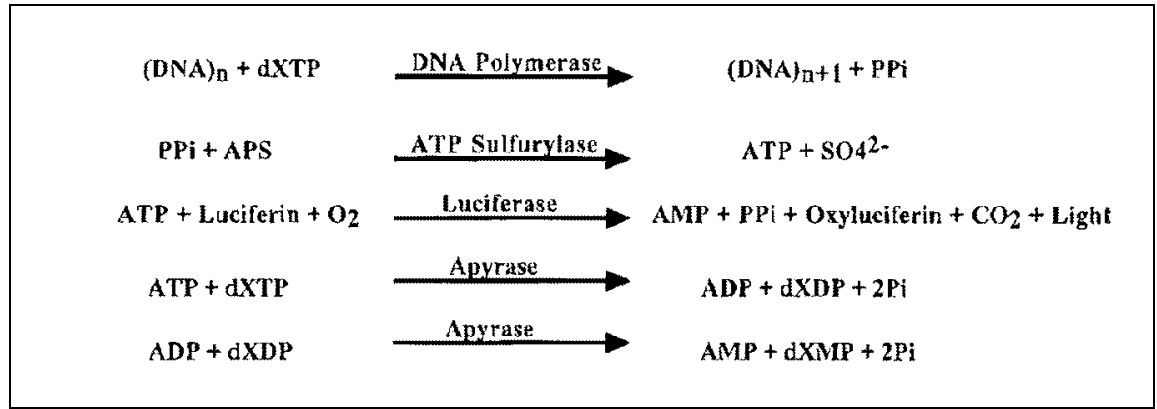

Figure 1. A schematic representation of the reactions in the pyrosequencing method. The four different nucleotides are added stepwise to the primed template. The $\mathrm{PP}_{\mathrm{i}}$ released in the DNA polymerasecatalyzed reaction is detected by the ATP sulfurylase and luciferase in a coupled reaction. The added nucleotides are continuously degraded by a nucleotide-degrading enzyme, apyrase. After the first added nucleotide has been degraded, the next nucleotide can be added. As this procedure is repeated, longer stretches of the template sequence are deduced. The dXTP indicates one of the four nucleotides, APS is adenosine phosphosulfate, and $\mathrm{n}$ is the number of residues.

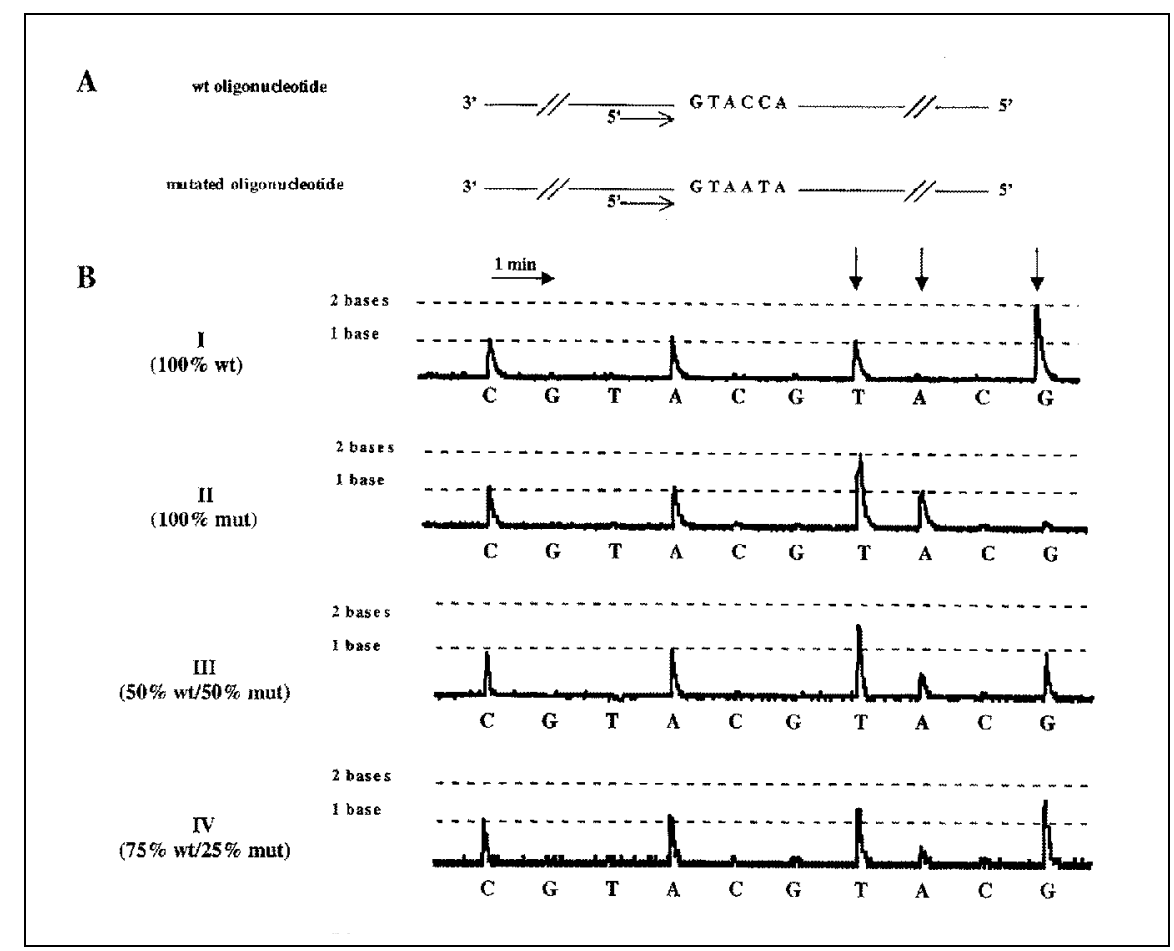

Figure 2. Determination of the detection limit of a mutation signal by pyrosequencing. (A) Two primed oligonucleotide templates representing the normal and the mutated alleles were used. (B) The height of the signals demonstrates the number of incorporated bases. The arrows indicate the positions where different ratios of the two oligonucleotide templates give rise to different signal levels (I-IV). A pattern difference can be observed between the $100 \%$ wild-type sequence and all mixture sequences and indicates the possibility of pattern recognition down to at least a $25 \%$ mutation signal. 
ming up the third (T), fourth (A) and fifth $(\mathrm{G})$ peak positions.

Different patterns indicate that the wild-type has one $\mathrm{T}$ followed by two Gs, but the mutated mixture has one and one-half $\mathrm{T}$, one-half $\mathrm{A}$ and one $\mathrm{G}$ instead. This accounts for an exact 50\% $\mathrm{CC}$ to TA mutations. A similar calculation can be made for the mixture that contains $25 \%$ mutant oligonucleotide. Furthermore, it is clear by comparing the wild-type sequence and the $25 \%$ mutant mixture that the limit of detection is approximately $25 \%$ mutant to wild-type signal.

\section{Conventional DNA Sequencing on Clinical Material}

To compare the use of pyrosequencing and conventional DNA sequencing on clinical material, we amplified the p53 tumor suppressor gene from microdissected tumor biopsies. We selected two samples, one from basal cell cancer and one from squamous cell cancer of the skin because these tumors frequently show multiple $p 53$ mutations within a single tumor lesion (9-11). Conventional DNA sequencing of the amplified material was primarily based on a dye primer chemistry protocol (1). Furthermore, these samples were negative for loss of heterozygosity using microsatellite markers (data not shown). DNA sequencing of the basal cell cancer sample revealed a double mutation (CC to TA) in exon 5. This mutation corresponds to codons 178 and 179, and the amino acid change is His-His to His-Asn. Furthermore, the mutant to wild-type signal was $50 \%$, which indicates that none or very few contaminating normal cells were present in the sample after microdissection (data not shown). DNA sequencing of the squamous cell cancer sample revealed two mutations within the same codon (codon 224) in exon 6. The mutation GAG to AAA gives the amino acid change Glu to Lys. In this case, the mutation signal was about $30 \%$ (data not shown). To investigate whether the mutations had occurred on the same or different alleles, cloning of the PCR products and re-sequencing of the clones was performed. In both cases, the mutations were shown to be located on the same allele (data not shown).

\section{Pyrosequencing on Clinical Material}

The procedure for pyrosequencing of the amplified basal cell cancer sample in exon 5 was the same as for the oligonucleotide model system. After hybridization of the sequencing primer to the ssDNA targets, iterative additions of nucleotides CGTA were performed and the output of light from the reaction was measured. Figure 3 demonstrates the result from pyrosequencing of the two compared tissues (normal/tumor). Figure 3B shows the raw data for the respective tissue, which clearly indicates a sequence difference between the two samples. Identical incorporations are achieved for the two first positions ( $\mathrm{C}$ and $\mathrm{A})$, but at the third position, the $\mathrm{T}$ nucleotide is incorporated at a higher degree in the tumor sequence.

In addition, the following sequencing peaks display additional pattern dissimilarities. The squamous cell cancer sample (Figure 4) with two mutations in the vicinity of exon 6 (GAG to AAA) was also tested in the same manner. As mentioned previously, about $60 \%$ of the microdissected cells contain a mutated p53 gene (30\% mutation signal, and mutation on only one allele) as determined by conventional DNA sequencing using labeled primers. Figure 4A outlines three fragments for both the normal sequence (three wild-type fragments) and for the tumor sequence (two wild-types and one mutated fragment) to demonstrate that the latter is a mixture of two types of fragments in a ratio of $2: 1$. The first signal in the raw data (Figure 4B) is obtained for incorporation of $\mathrm{T}$ in both samples. Since one base incorporation represents three different fragments in the sample, the signal for incorporated bases has been divided into three. The addition of A does not give rise to any incorporation in the normal sample, but the incorporation of three As occurs in one-third of the fragments in the tumor sample. These three A residues arise from the population of the mutated fragment while the wildtype fragments remain unextended. Therefore, a non-synchronized exten-

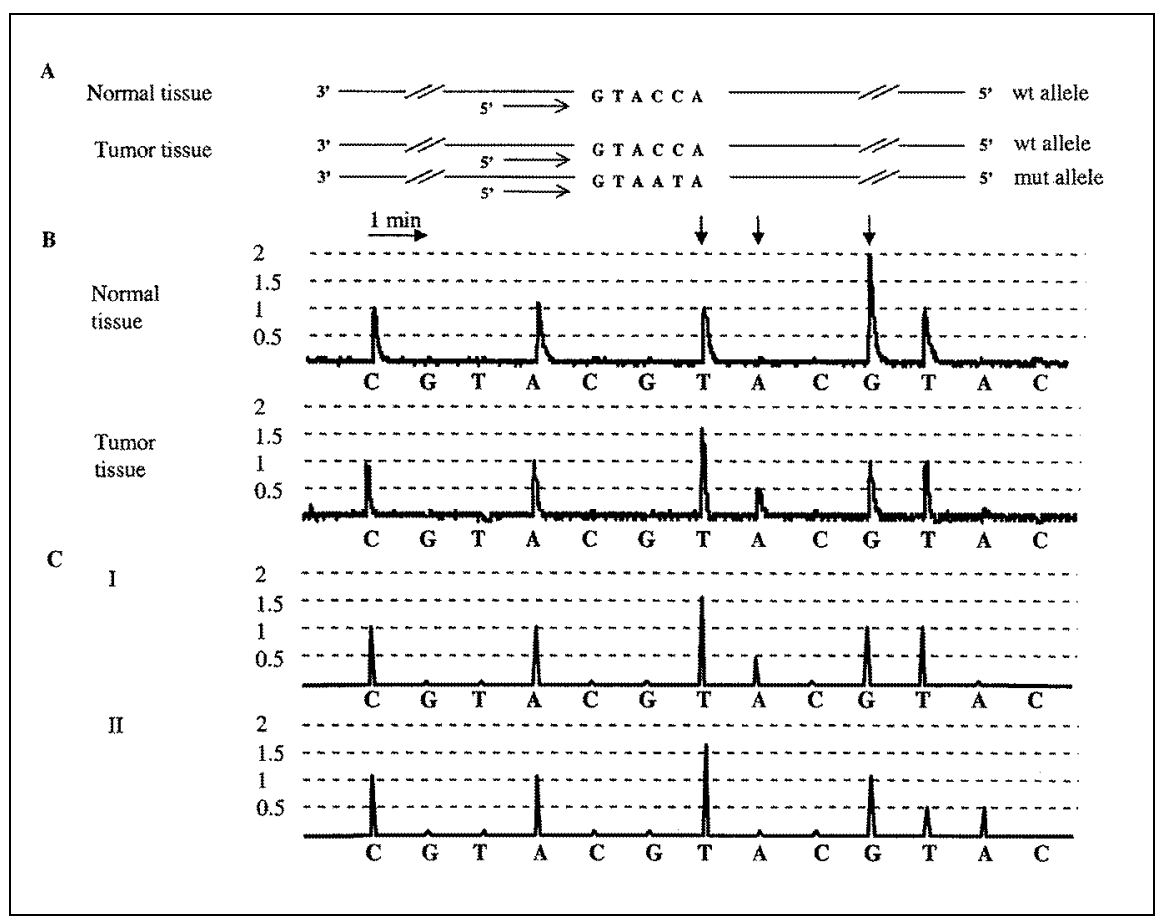

Figure 3. Traces demonstrating allelic determination from the polymorphic region in exon 5 of the p53 gene. (A) The sequences of normal and tumor tissues are indicated. (B) The lower panel demonstrates occurrence of $50 \%$ double mutations as the 1.5 -base signal for $\mathrm{T}$ with subsequent 0.5 -base signal for $\mathrm{A}$ and 1-base signal for $\mathrm{G}$ is obtained. The result clearly demonstrates that both mutations reside on the same allele while the nucleotide extension can be followed on the separate alleles. (C) A schematic representation demonstrating the expected pyrosequencing patterns in the sequence of exon 5 when the mutations reside on the same allele (C, I) or on different alleles (C, II). 
sion will be observed during the sequencing of the tumor sample. As two phases of nucleotide incorporation on different alleles are obtained in these cases, pyrosequencing enables determination of biallelic mutations. Figures $3 \mathrm{C}$ and $4 \mathrm{C}$ show schematically expected patterns when the mutations reside on the same (I) or on different alleles (II) of the $p 53$ gene. A comparison of raw data (Figures 3B and 4B) with the expected patterns in Figures 3C and 4C clearly shows that, in both cases, the mutations have occurred on the same allele.

\section{DISCUSSION}

The objective of the study was to investigate the feasibility of using pyrosequencing for reading through polymorphic sequences and to determine the detection limit of mutations. We looked for mutations in the $p 53$ tumor suppressor gene and compared our results with conventional DNA sequencing. The most conspicuous difference in detecting polymorphism with the two methods is the raw data. A point mutation analyzed by conventional DNA sequencing will yield a change in one position, while a complete change of pattern is obtained when pyrosequencing is employed (Figures 3 and 4).

There are several technical advantages in using pyrosequencing for a reading of the polymorphic region. First, in the case of multiple mutations such as for $p 53$ in non-melanoma skin cancers, this technique determines the allele on which these mutations reside, without performing cloning experiments. In the cases discussed here, the mutations were near each other. Theoretically, it will be possible to follow the sequence of a single allele if the order of nucleotide additions is designed to enable nonsynchronized extension in the two alleles. Thus, an allelic determination of mutations that are far from each other can be performed. Second, the mutation signals can be quantified. Here, we show that a $25 \%$ mutation signal can be detected (Figure 2). Third, pyrosequencing bypasses the problem with DNA impurity, such as the appearance of truncated PCR products in the system, which are usually the result of nonspecific extension in the PCR. A low

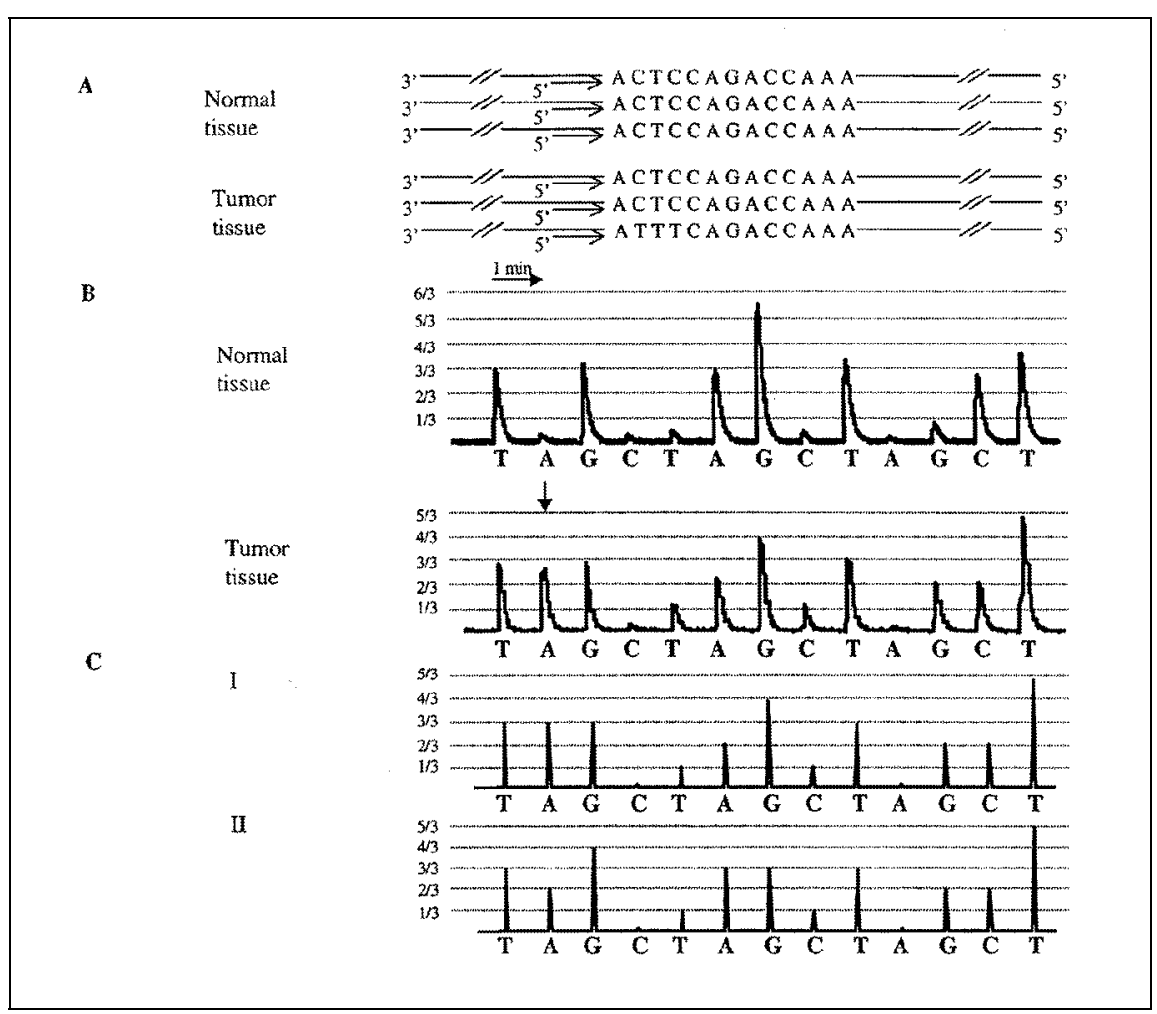

Figure 4. Allelic determination by pyrosequencing on tumor tissue with double mutations (GAG to AAA) in exon 6 of the p53 gene. (A) Tumor tissue, which is heterogeneous, contains sequences from normal and mutated fragments as presented by three possible sequences. (B) The obtained results by pyrosequencing determine that the mutations reside on the same allele as the nucleotide extension can be followed on the separate alleles. (B) The result in the lower panel can be compared to the obtained data on the normal template as shown in the top panel. The arrow indicates the base position in which the non-synchronized extension of a different allele is obtained. (C) Lane I and II is a schematic representation demonstrating the expected pyrosequencing patterns if the mutations reside on the same allele (I) or on different alleles (II), respectively.

amount of these products results in runoff signals in dye-primer conventional DNA sequencing, which gives rise to a strong signal covering the flanking basespecific signals (16). This signal then makes it impossible to determine the sequence in the region. Here, this problem actually occurred for the basal cell cancer sample in exon 5 (data not shown), while the pyrosequencing protocol tolerates the occurrence of truncated PCR products as we could read through the mutated region (Figure 3).

One parameter for consideration is the order of nucleotide addition in certain cases of multiple mutations for the determination of biallelic alterations. Although the polymorphisms can be detected regardless of the order of nucleotide addition, synchronized extensions on both alleles in a reaction may cause difficulties in interpretating the obtained data. For example, in Figure 4C, I and II, the order of nucleotide addition is TAGC and a clear pattern difference will be obtained when the adjacent mutations are placed on the same allele (Figure 4C, I) or different alleles (Figure 4C, II). On the other hand, an additional order of TGAC instead of TAGC would read through the mutations with no pattern difference (data not shown).

In conclusion, we show the possibility of using the pyrosequencing technique for allelic mutation analysis. The results presented also show the technique as a potentially powerful tool for resequencing of disease genes, the determination of heteroplasmy in mitochondrial DNA and the characterization of single nucleotide polymorphisms in a relatively short time. A recent fully automated system now allows parallel processing of 96 samples simultaneously, which offers a tremendous reduction in time for large-scale analysis. 


\section{REFERENCES}

1.Berg, C., A. Hedrum, A. Holmberg, F. Pontén, M. Uhlén and J. Lundeberg. 1995. Direct solid-phase sequence analysis of the human $p 53$ gene by use of multiplex polymerase chain reaction and alpha-thiotriphosphate nucleotides. Clin. Chem. 41:1461-1466.

2.Fearon, E.R. and B. Vogelstein. 1990. A genetic model for colorectal tumorigenesis. Cell 61:759-767.

3.Harris, C.C. 1993. p53: at the crossroads of molecular carcinogenesis and risk assessment. Science 262:1980-1981.

4.Hedrum, A., F. Pontén, Z. Ren, J. Lundeberg, J. Pontén and M. Uhlén. 1994. Sequence-based analysis of the human $p 53$ gene based on microdissection of tumor biopsy samples. BioTechniques 17:118-129.

5.Hollstein, M., D. Sidransky, B. Vogelstein and C.C. Harris. 1991. p53 mutations in human cancers. Science 253:49-53.

6.Hultman, T., S. Ståhl, E. Hornes and M. Uhlén. 1989. Direct solid phase sequencing of genomic and plasmid DNA using magnetic beads as solid support. Nucleic Acids. Res. 17:4937-4946.

7.Karamohamed, S., J. Nilsson, K. Nourizad, M. Ronaghi, B. Pettersson and P. Nyrén.
1999. Production, purification, and luminometric analysis of recombinant saccharomyces cerevisiae MET3 adenosine triphosphate sulfurylase expressed in Escherichia coli. Protein Expr. Purif. 15:381-388.

8.Knudson, A.G., Jr. 1971. Mutation and cancer: statistical study of retinoblastoma. Proc. Natl. Acad. Sci. USA. 68:820-823.

9.Pontén, F., C. Berg, A. Ahmadian, M. Nister, J. Lundeberg, M. Uhlén and J. Pontén. 1997. Molecular pathology in basal cell cancer with $p 53$ as a genetic marker. Oncogene 15:1059-1067.

10.Ren, Z.P., A. Ahmadian, F. Pont,n, M. Nister, C. Berg, J. Lundeberg, M. Uhlén and J. Pontén. 1997. Benign clonal keratinocyte patches with $p 53$ mutations show no genetic link to synchronous squamous cell precancer or cancer in human skin. Am. J. Pathol. 150:1791-1803.

11.Ren, Z.P., A. Hedrum, F. Pontén, M. Nister, A. Ahmadian, J. Lundeberg, M. Uhlén and J. Pontén. 1996. Human epidermal cancer and accompanying precursors have identical p53 mutations different from $p 53$ mutations in adjacent areas of clonally expanded non-neoplastic keratinocytes. Oncogene 12:765-773.

12.Ronaghi, M., M. Nygren, J. Lundeberg and P. Nyrén. 1999. Analyses of secondary struc- tures in DNA by pyrosequencing. Anal. Biochem. 267:65-71.

13.Ronaghi, M., B. Pettersson, M. Uhlén and P. Nyrén. 1998. PCR-introduced loop structure as primer in DNA sequencing. BioTechniques 25:876-884.

14.Ronaghi, M., M. Uhlén and P. Nyrén. 1998. A sequencing method based on real-time pyrophosphate. Science 281:363, 365.

15.Satake, N., S. Urakami, Y. Hirayama, K. Izumi and O. Hino. 1998. Biallelic mutations of the Tsc2 gene in chemically induced rat renal cell carcinoma. Int. J. Cancer 77:895-900.

16.Westberg, J., A. Holmberg, M. Uhlén and B. Pettersson. 1999. Truncated fragments in polymerase chain reaction-based DNA sequencing. Electrophoresis 20:502-510.

Received 15 June 1999; accepted 20 August 1999.

\section{Address correspondence to:}

Dr. Mostafa Ronaghi

DNA Sequencing and Technology Center

Stanford University

855 California Avenue

Palo Alto, CA 94304, USA

Internet:mostafa@sequence.stanford.edu 